\title{
Redução do erro amostral na estimativa do volume de povoamentos de Eucalyptus ssp. por meio de escaneamento laser aerotransportado
}

\author{
Reducing of the sampling error in Eucalyptus ssp. stand \\ volume through airborne laser scanning estimation
}

\author{
Danitiele Cristina França Laranja ${ }^{1}$, Eric Bastos Gorgens ${ }^{2}$, Carlos Pedro Boechat Soares ${ }^{3}$, \\ André Gracioso Peres da Silva ${ }^{4}$ e Luiz Carlos Estraviz Rodriguez ${ }^{5}$
}

\begin{abstract}
Resumo
O inventário florestal é uma importante atividade que fornece uma ampla gama de informações sobre os parâmetros populacionais de extensas áreas florestais, com base em delineamentos de amostragem. Assim, este trabalho apresenta e avalia o uso combinado de levantamentos laser aerotransporto (ALS - Airborne Laser Scanning) e de dupla amostragem para estimativa de volume em plantios de eucalipto, comparando os resultados obtidos com delineamentos amostrais tradicionais como a amostragem casual simples e a amostragem casual estratificada. No primeiro cenário de dupla amostragem assumiu-se a primeira fase como sendo a métrica de altura percentil 90 (P90) derivado dos dados ALS. No segundo cenário, adotou-se o percentil 90 (P90) e a métrica de proporção porcentagem de todos os retornos acima da média relacionada aos primeiros retornos (ARMFR), derivadas dos dados LiDAR (Light Detection And Ranging). Através de simulações buscou-se determinar a intensidade amostral necessária para atender um erro de amostragem máximo de $5 \%$. O estudo foi realizado em uma área de 401,6 hectares localizada nos municípios de São Miguel Arcanjo e Pilar do Sul, onde foram lançadas e medidas 37 parcelas em campo. O menor erro de amostragem obtido foi o da dupla amostragem com regressão múltipla $( \pm 1,8 \%)$, seguidos pela dupla amostragem com estimador de regressão simples; amostragem casual estratificada; e amostragem casual simples, confirmando o potencial de uso dos dados do sensor laser para melhorar as estimativas de volume do inventário florestal, possibilitando a redução da intensidade amostral. Considerando apenas 10 unidades de amostra, o erro da dupla amostragem obtido na regressão múltipla foi de $\pm 3,4 \%$.
\end{abstract}

Palavras-chave: LiDAR, Delineamento Amostral, Erro Amostral, Dupla Amostragem, Métricas ALS.

\begin{abstract}
Forest inventory is an important activity to provide a wide range of information about the parameters from extensive forested areas based on sampling designs. This study evaluates the use of ALS (Airborne Laser Scanning) metrics as part of a double sampling design for stand volume estimation in Eucalyptus plantation, comparing the results to traditional sampling designs such as simple random sampling and stratified random sampling. In one scheme, the double sampling first phase was the height metric of the $90^{\text {th }}$ percentile (P90) derived from LiDAR (Light Detection And Ranging) data. In the second double sampling scenario we adopted the 90th percentile (P90) and the density metric percentage of all returns above the mean of first returns (ARMFR), also derived from LiDAR data. Through simulations we sought to determine the sampling intensity required to obtain a sampling error at most $5 \%$. The study was conducted in 401,6 hectares of Eucalyptus plantation located between the municipalities of São Miguel Arcanjo and Pilar do Sul (São Paulo), where 37 plots were measured in the field. The smallest sampling error was obtained from the double sampling with multiple regression $( \pm 1,8 \%)$ followed by the double sampling with simple regression, the stratified random sampling and the simple random sampling; confirming the use of ALS data to improve the volume estimation and enabling the sampling intensity reduction. The error of double sampling with multiple regression was $\pm 3,4 \%$ considering only 10 field plots.
\end{abstract}

Keywords: LiDAR, double sampling, sample design, sampling error, ALS metrics.

\footnotetext{
${ }^{1}$ Mestranda em Recursos Florestais. USP - Universidade de São Paulo - Departamento de Ciências Florestais / ESALQ - Escola Superior de Agricultura "Luiz de Queiroz". Av. Pádua Dias, 11 - 13418-900 - Piracicaba, SP. E-mail: danitiele.cfl@gmail.com. 2Professor e Pesquisador do Departamento de Engenharia Florestal. UFVJM - Universidade Federal dos Vales do Jequitinhonha e Mucuri. Campus JK - Rod. MGT 367, Km 583, 5000 - Alto do Jacuba - 39100-000 - Diamantina, MG. E-mail: gorgens@usp.br.

3Professor Titular do Departamento de Engenharia Florestal. UFV - Universidade Federal de Viçosa - Centro de Ciências Agrárias - Campus Universitário - 36570-900 - Viçosa, MG. E-mail: csoares@ufv.br.

${ }^{4}$ Mestre em Recursos Florestais. USP - Universidade de São Paulo - Departamento de Ciências Florestais / ESALQ - Escola Superior de Agricultura "Luiz de Queiroz". Av. Pádua Dias, 11 - 13418-900 - Piracicaba, SP. E-mail: andregracioso@gmail.com. ${ }^{5}$ Professor Associado do Departamento de Ciências Florestais. USP - Universidade de São Paulo / ESALQ - Escola Superior de Agricultura "Luiz de Queiroz". Av. Pádua Dias, 11 - 13418-900 - Piracicaba, SP. E-mail: lcer@usp.br.
}

Sci. For., Piracicaba, v. 43, n. 108, p. 845-852, dez. 2015 DOI: dx.doi.org/10.18671/scifor.v43n108.9 
Laranja et al. - Redução do erro amostral na estimativa do volume de povoamentos

de Eucalyptus ssp. por meio de escaneamento laser aerotransportado

\section{INTRODUÇÃO}

O Inventário florestal tem por objetivo a quantificação e qualificação dos atributos florestais existentes em uma área, sendo uma peça importante dentro do manejo florestal, seja de florestas naturais, seja de florestas plantadas. Ele é capaz de fornecer detalhes da floresta, por meio de técnicas de amostragem que estimam os parâmetros da população (PÉLLICO NETTO; BRENA, 1997; MORAIS FILHO et al., 2003).

Em função do tamanho das florestas e da densidade de indivíduos por unidade de área, normalmente os inventários florestais são realizados por meio de amostragem. Diferentes delineamentos amostrais existem para melhor estimar o valor de um determinado parâmetro populacional e a sua variação. O erro amostral $(E(\%))$ é determinado a partir do erro padrão da média $\left(S_{\bar{y}}\right)$, que nada mais é que uma medida de variabilidade da média quando repetidas amostras são tomadas da população (SHIVER; BORDERS, 1996).

Na amostragem casual simples (ACS), todos os elementos da população possuem a mesma probabilidade de pertencer à amostra. A principal limitação da ACS é que o lançamento aleatório das parcelas pode resultar na não representação de todos os estratos da floresta, resultando assim em estimativas imprecisas da população. Neste caso, quando a população pode ser dividida em estratos homogêneos, emprega-se a amostragem casual estratificada (ACE). Os estratos devem ser representados na amostra na mesma proporção com que existem na população. Quanto mais estratificada a floresta e consequentemente mais homogêneo os estratos, o erro amostral tende a diminuir, uma vez que o erro padrão da média também diminui.

Em casos em que um maior nível de detalhe e precisão são requeridos, a dupla amostragem (ou amostragem em duas fases) se oferece como uma interessante alternativa. Na primeira fase (também conhecida como fase simples), os esforços são direcionados para a obtenção de uma variável auxiliar, sem se preocupar com a variável de interesse. Assim, a variável auxiliar deve ser de mais fácil mensuração e altamente correlacionada com a variável de interesse. A intensidade amostrada durante a primeira fase é geralmente superior à intensidade da segunda fase (SHIVER; BORDERS, 1996).

Na segunda fase (também conhecida como fase completa), a intensidade amostral é menor e medem-se simultaneamente a variável auxiliar e a variável de interesse nas unidades amostrais. Não é necessário que as fases ocorram de maneira simultânea. No entanto, é fundamental que na segunda fase sejam coletadas as duas variáveis de forma a permitir que um estimador de regressão relacione a variável auxiliar e a variável de interesse (SHIVER; BORDERS, 1996).

A dupla amostragem (DA) permite que as informações dos dados auxiliares sejam usadas para melhorar a precisão e exatidão das estimativas das variáveis do inventário florestal, quando comparadas com os inventários baseados apenas nas observações da variável de interesse. Essa melhoria é obtida através do uso de estimadores de regressão ou de razão e depende do nível de correlação entre os dados de sensoriamento remoto e as variáveis de inventário florestal (STEPHENS et al., 2012).

É muito comum o uso de informações obtidas por sensores remotos na primeira fase de uma dupla amostragem em levantamentos florestais. Andersen e Breidenbach (2007), por exemplo, demonstraram que o emprego de variáveis auxiliares obtidas a partir do sensoriamento remoto pode aumentar a precisão e a exatidão das estimativas de inventário. As primeiras aplicações da dupla amostragem em inventários florestais utilizaram na primeira fase informações extraídas de fotos aéreas. Em seguida, imagens de satélite passaram a substituir as fotos aéreas (VAN DEUSEN et al., 1999). O escaneamento laser aerotransportado, baseado em tecnologias LiDAR (Light Detection And Ranging) apresenta-se como alternativa tecnológica viável capaz de produzir bons resultados quando métricas resultantes desses levantamentos são usadas como variáveis auxiliares em inventários de dupla amostragem.

O escaneamento laser aerotransportado (ALS - Airborne Laser Scanning) tem ganhado destaque (MALTAMO et al., 2004). Os sistemas LiDAR, emissores de pulsos de luz laser, baseiam-se nos mesmos princípios dos sistemas RADAR, emissores de ondas de rádio (GIONGO et al., 2010). A tecnologia LiDAR baseada em sensores acoplados aos processos de varredura a laser aerotransportado gera uma grande quantidade informação tridimensional sobre a estrutura vertical e horizontal da floresta com alta precisão, baixo custo e alta velocidade de aquisição de dados (GIONGO et al., 2010). 
A escolha do melhor método amostral, além de considerar as características da população, deve adequar a expectativa de maior precisão ao menor custo possível. A não consideração de características específicas da população estudada, pode acarretar custos maiores de inventário (CESARO et al., 1994). Dessa forma, justificam-se as análises de diferentes métodos de amostragem, que busquem reduzir o tempo e o custo de execução do inventário, sem que haja prejuízos à precisão e à exatidão das estimativas.

Næsset et al. (2013) reforçam a recomendação de desenhos amostrais eficientes para inventários, especialmente relacionados à incerteza e aos custos. Diferentes estratégias de amostragem têm sido analisadas com base em técnicas de simulação.

A hipótese deste trabalho é de que a alta densidade de pontos amostrados em levantamentos laser aerotransportados aliado à alta correlação entre as métricas LiDAR e parâmetros biofísicos da floresta pode ser utilizada na primeira fase do método dupla amostragem e, também, para reduzir esforço e intensidade amostral de campo.

\section{MATERIAL E MÉTODOS}

O estudo foi realizado em uma área de 401,6 hectares pertencente à empresa Suzano Papel e Celulose, localizada no interior do Estado de São Paulo, nos municípios de São Miguel Arcanjo e Pilar do Sul (Figura 1). O clima da região é caracterizado pela precipitação média anual de 1400 mm e altitude média de 715 m (classificação de Köppen: Cfb). O relevo é ondulado com inclinação máxima de 32 graus (GAVA, 1997).

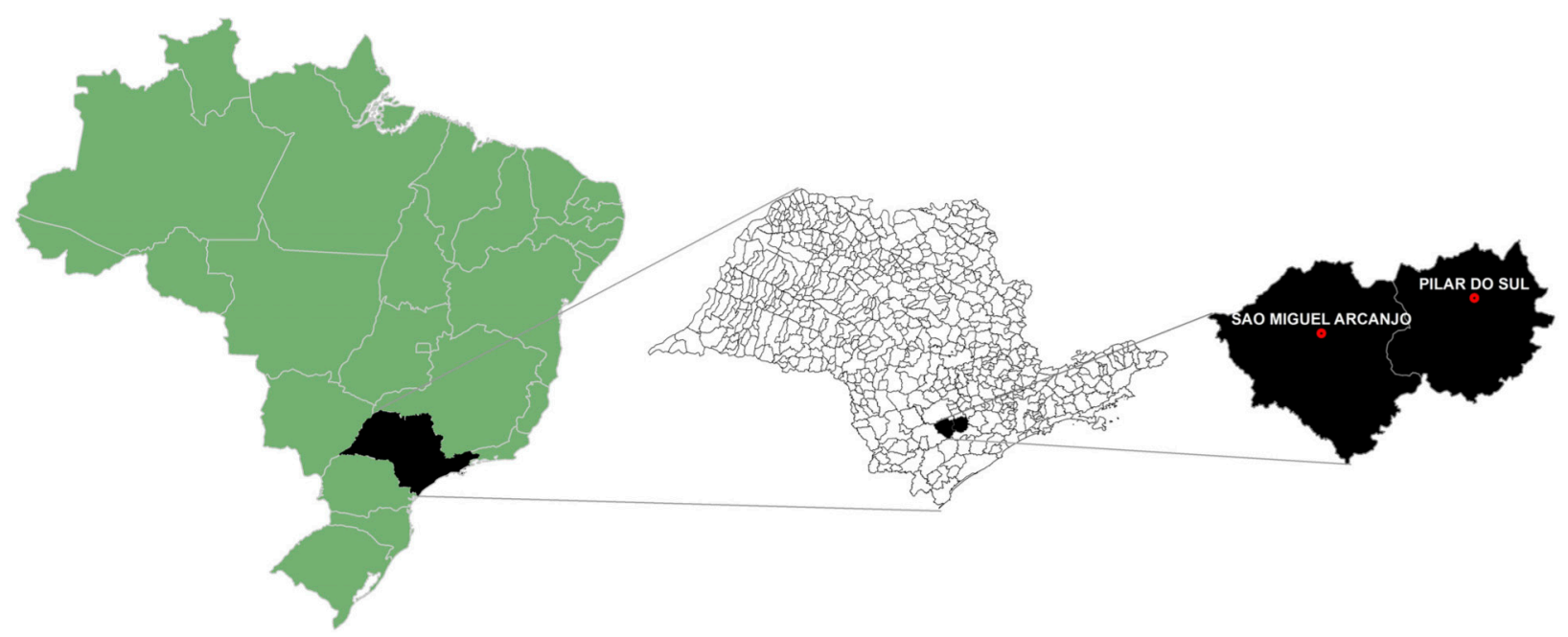

Figura 1. Localização dos municípios da área de estudo.

Figure 1. Localization of the study area.

A área é dividida em talhões, formados por plantios clonais do gênero Eucalyptus, manejados para a produção de celulose. Apresentam idade de seis anos e foram plantados utilizando-se clones originários de quatro grupos genéticos diferentes, em espaçamento $3 \times 2,2 \mathrm{~m}$. Foram considerados neste estudo os dados coletados em 37 parcelas circulares com área de 380,13 m² (Tabela 1).

Tabela 1. Caracterização dos povoamentos do estudo.

Table 1. Characterization of the study stands.

\begin{tabular}{|c|c|c|c|c|c|c|c|c|}
\hline \multirow{2}{*}{$\begin{array}{l}\text { Unidade de } \\
\text { Produção }\end{array}$} & \multirow{2}{*}{ Área (ha) } & \multirow{2}{*}{$\mathbf{N}^{\circ}$ de parcelas } & \multicolumn{2}{|c|}{ DAP (cm) } & \multicolumn{2}{|c|}{ Altura (m) } & \multicolumn{2}{|c|}{ VTCC $\left(\mathrm{m}^{3} / \mathrm{ha}\right)$} \\
\hline & & & Média & Desvio & Média & Desvio & Média & Desvio \\
\hline 1 & 101,47 & 10 & 14,0 & 0,61 & 23,2 & 0,86 & 266,5 & 30,15 \\
\hline 2 & 64,18 & 5 & 14,8 & 0,38 & 24,8 & 0,62 & 293,7 & 31,22 \\
\hline 3 & 50,32 & 5 & 15,9 & 0,27 & 25,9 & 0,65 & 358,4 & 25,24 \\
\hline 4 & 83,31 & 7 & 16,0 & 0,50 & 26,6 & 0,61 & 387,7 & 25,36 \\
\hline 5 & 102,34 & 10 & 16,2 & 0,88 & 26,3 & 1,07 & 362,9 & 42,87 \\
\hline
\end{tabular}

Obs.: DAP = Diâmetro a altura do peito, VTCC $=$ Volume total com casca. 
Laranja et al. - Redução do erro amostral na estimativa do volume de povoamentos

de Eucalyptus ssp. por meio de escaneamento laser aerotransportado

\section{Levantamento dos dados LiDAR}

Os dados LiDAR foram obtidos em dezembro de 2013, com uma aeronave CESNA modelo 206, equipada com uma unidade inercial Applanix (IMU +GPS) modelo POS AV 150 e sensor LiDAR Harrier 68i da Trimble. As especificações do voo encontram-se detalhadas na Tabela 2.

Tabela 2. Especificações do voo.

Table 2. Flight specifications.

\begin{tabular}{lc}
\hline Características & Descrição dos parâmetros \\
\hline Densidade de pontos & 10 pontos $/ \mathrm{m}^{2}$ \\
Diâmetro do pulso no alvo (footprint) & $0,22 \mathrm{~m}$ \\
Ângulo de abertura para varredura (FOV) & $45^{\circ}$ \\
Faixa de varredura & $363,11 \mathrm{~m}$ \\
Altura do voo & $438,32 \mathrm{~m}$ \\
Taxa de escaneamento & $174,05 \mathrm{~Hz}$ \\
Taxa de emissão & $400 \mathrm{kHz}$ \\
\hline
\end{tabular}

\section{Levantamento de campo}

As 37 parcelas foram lançadas de forma aleatória dentro das 5 unidades de produção (UP). Em cada parcela foram medidos os diâmetros à altura do peito (DAP) de todas as árvores, a altura total das 10 árvores localizadas na linha central da parcela e 4 alturas totais das árvores de maior DAP. O modelo hipsométrico de Campos et al. (1984) foi utilizado para estimação das alturas (H) das árvores não medidas e o volume foi estimado pelo modelo de Schumacher e Hall (1933), para os quais foram fornecidos pela empresa os coeficientes adequados para a região onde o estudo está inserido. O volume total com casca (VTCC) de cada parcela é resultado da soma dos volumes individuais, e convertido para volume por hectare com base na área da parcela.

\section{Delineamentos amostrais}

O volume total com casca da área de estudo foi estimado através de três delineamentos amostrais consolidados em cinco cenários. No primeiro, as parcelas foram processadas assumindo amostragem casual simples (ACS). Em seguida, duas estratificações foram implementadas. A primeira amostragem casual estratificada (ACE I) usou apenas o material genético como variável de estratificação. Na ACE II considerou-se o material genético e a unidade de produção (UP) como critérios de estratificação. A UP foi escolhida por agrupar áreas submetidas a condições semelhantes de manejo. Por fim, duas abordagens de dupla amostragem (DA) pelo estimador da regressão foram desenvolvidas. A primeira abordagem (DA I) assumiu apenas uma métrica LiDAR como variável auxiliar na primeira fase. A segunda abordagem (DA II) considerou duas métricas LiDAR como variáveis auxiliares na primeira fase. As métricas usadas como variáveis auxiliares expressam estatísticas derivadas da distribuição vertical dos retornos LiDAR (SILVA et al., 2015).

$\mathrm{Na}$ amostragem casual simples, sendo $\bar{Y}$ a média amostral e $t$ a estatística t de Student, tabelada para $n-1$ graus de liberdade com 5\% de significância, o erro de amostragem percentual pode ser estimado pela equação 1 (SHIVER; BORDERS, 1996).

$$
E(\%)=\frac{s_{\bar{Y}} \cdot t}{\bar{Y}} \cdot 100 \text { (1) }
$$

Ainda com relação à ACS, sendo $\gamma_{i}$ o volume por hectare da $i$-ésima parcela, $S_{\gamma}^{2}$ é a variância do volume por hectare das parcelas, $n$ o número de parcelas e $N$ é o número total de unidades amostrais possíveis dentro da população, a média (Equação 2) e a variância da média (Equação 3 ) podem ser calculadas da seguinte forma (SHIVER; BORDERS, 1996):

$$
\begin{array}{r}
\bar{Y}=\frac{\sum y_{i}}{n} \\
S_{\bar{y}}^{2}=\frac{S_{y}^{2}}{n} \cdot\left(\frac{N-n}{N}\right)
\end{array}
$$

O erro padrão da média pode ser então calculado através da equação 4:

$$
S_{\bar{y}}= \pm \sqrt{S_{\bar{y}}^{2}}
$$


Na amostragem casual estratificada, sendo $\bar{Y}_{j}$ a média dos volumes das unidades de amostra do j-ésimo estrato, $n_{j}$ o número de parcelas no j-ésimo estrato, $N_{j}$ o número de parcelas possível no j-ésimo estrato, $N$ o número de unidades de amostra possíveis na população e $S^{2}$ a variância do $j$ -ésimo estrato, a média estratificada (Equação 5) e a variância da média estratificada (Equação 6) podem ser estimadas através de (SHIVER; BORDERS, 1996):

$$
\begin{gathered}
\bar{Y}=\frac{\sum N_{j} \bar{Y}_{j}}{N} \\
S_{\bar{y}}^{2}=\sum\left(\left(\frac{N_{j}}{N}\right)^{2} \cdot \frac{S_{j}^{2}}{n_{j}} \cdot\left(\frac{N_{j}-n_{j}}{N_{j}}\right)\right)
\end{gathered}
$$

O erro padrão da média foi obtido, extraindo-se a raiz quadrada da equação 6 .

Na DA I, o estimador da regressão (Equação 7) foi escolhido para relacionar a variável de interesse ( $2^{\text {a }}$ fase) volume total com casca (VTCC) com a variável auxiliar ( $1^{\text {a }}$ fase) percentil 90 (P90), que expressa a altura abaixo da qual se encontram 90\% dos retornos LiDAR:

$$
\text { VTCC }=\beta_{0}+\beta_{1} P 90+\varepsilon
$$

Em que $\beta_{i}=$ coeficientes do modelo e $\varepsilon=$ erro aleatório.

Já na DA II, foram utilizadas duas variáveis auxiliares (percentil 90 - P90 e porcentagem de retornos acima da média dos primeiros retornos - ARMFR) em uma regressão linear múltipla (Equação 8) para relacionar essas duas métricas LiDAR ( $1^{\text {a }}$ fase) com a variável de interesse VTCC ( $2^{\text {a }}$ fase).

$$
V T C C=\beta_{0}+\beta_{1} P 90+\beta_{2} \text { ARMFR }+\varepsilon
$$

Em que $\beta_{i}=$ coeficientes do modelo e $\varepsilon=$ erro aleatório.

Na dupla amostragem, a variância da média amostral (Equação 9) é uma combinação da variância do VTCC mensurado na $2^{\mathrm{a}}$ fase e do estimador da regressão que relaciona a variável auxiliar com a variável de interesse (Equação 10), podendo ser estimado por (SHIVER; BORDERS, 1996):

$$
S_{\bar{y}_{d l}}^{2}=\frac{S_{y}^{2}}{n} \cdot\left(1-\left(\frac{n^{\prime}-n}{n^{\prime}}\right) \cdot \rho^{2}\right)
$$

Em que $n^{\prime}$ e $n=$ número de parcelas na $1^{\mathrm{a}}$ e $2^{\mathrm{a}}$ fase, respectivamente e $\rho$ pode ser calculado a partir do coeficiente de determinação ajustado do modelo (SHIVER; BORDERS, 1996):

$$
\rho=\sqrt{R_{a d j}^{2}} \quad(10)
$$

A métrica de altura P90 foi escolhida com base no trabalho desenvolvido por Zonete et al. (2010), que encontrou o percentil 90 (P90) entre as métricas mais promissoras para modelar volume de plantios florestais. Silva et al. (2015) sugeriu a inclusão métricas de proporção para ajudar a explicar parte da variação do volume. Assim na DA II, foi adicionado ao estimador da regressão a métrica de densidade ARMFR.

\section{Intensidade amostral}

Para que a determinação da intensidade amostral ideal nas cinco estratégias amostrais se faça de forma comparativa optou-se por utilizar um processo de simulação variando gradativamente o número de parcelas até que o erro amostral atinja nível satisfatório.

O processo de simulação consistiu, portanto, num processo de redução gradativa ao acaso do número de parcelas $(n)$ até que o erro amostral médio atingisse $\pm 5 \%$. A cada redução, $n$ parcelas eram escolhidas ao acaso e as estatísticas da amostragem (erro padrão, intervalo de confiança e erro amostral) recalculadas. O processo de escolha e de cálculo das estatísticas da amostragem foi repetido 50 vezes para cada redução. Caso a redução atingisse o número mínimo de parcelas definido para cada delineamento amostral, sem que o erro atingisse $\pm 5 \%$, a simulação era encerrada. $O$ número mínimo de parcelas foi definido como 10 parcelas para as amostragens ACS e DA, e em 3 parcelas por estrato na amostragem ACE. 
Laranja et al. - Redução do erro amostral na estimativa do volume de povoamentos

de Eucalyptus ssp. por meio de escaneamento laser aerotransportado

\section{RESULTADOS E DISCUSSÃO}

A estratificação reduziu o erro amostral em mais de 2 pontos percentuais. Enquanto que o erro via amostragem casual simples apresentou um erro amostral de $\pm 4,8 \%$, a estratificação por um nível (material genético) apresentou um erro amostral de $\pm 3,4 \%$, que pôde ser reduzido para $\pm 2,6 \%$ quando a amostragem foi implementada em dois níveis (material genético e UP). Essa melhora era esperada, uma vez que a estratificação aumenta a homogeneidade das sub amostras e reduz a variância global (Tabela 3).

Tabela 3. Resumo das estatísticas dos delineamentos amostrais.

Table 3. Summary of the statistics of the sampling designs.

\begin{tabular}{lccccc}
\hline Amostragem & $\begin{array}{c}\text { VTCC } \\
\left(\mathbf{m}^{\mathbf{3}} / \mathbf{h a}\right)\end{array}$ & $\begin{array}{c}\text { Variância da média } \\
\left(\mathbf{m}^{\mathbf{3}} \mathbf{h a}\right)\end{array}$ & $\begin{array}{c}\text { Erro Padrão } \\
\left(\mathbf{m}^{\mathbf{3}} / \mathbf{h a}\right)\end{array}$ & $\begin{array}{c}\text { Intervalo de Confiança } \\
\left(\mathbf{m}^{\mathbf{3}} \mathbf{h} \mathbf{h a}\right)\end{array}$ & $\begin{array}{c}\text { Erro Amostral } \\
(\%)\end{array}$ \\
\hline ACS & 331,6 & 89,7 & $\pm 9,5$ & $\pm 16,0$ & $\pm 4,8$ \\
ACE I & 335,5 & 45,3 & $\pm 6,7$ & $\pm 11,4$ & $\pm 3,4$ \\
ACE II & 333,3 & 27,0 & $\pm 5,2$ & $\pm 8,8$ & $\pm 2,6$ \\
DA I & 319,8 & 14,5 & $\pm 3,8$ & $\pm 6,4$ & $\pm 2,0$ \\
DA II & 328,3 & 12,8 & $\pm 3,6$ & $\pm 6,0$ & $\pm 1,8$ \\
\hline
\end{tabular}

A dupla amostragem atingiu um erro amostral ainda menor, chegando a $\pm 2,0 \%$ utilizando uma regressão simples como estimador (DA I) e a $\pm 1,8 \%$ utilizando a regressão múltipla como estimador (DA II). A regressão linear simples (Equação 7) apresentou coeficientes significativos (Tabela 4), coeficiente de determinação ajustado ( $\left.\mathrm{R}^{2} \mathrm{adj}\right)$ de 83,7 \% e erro padrão dos resíduos de 23,3 ( $\mathrm{m}^{3} / \mathrm{ha}$ ). A análise de resíduos pode ser visualizada na Figura 2.

Tabela 4. Resumo estatístico da regressão linear simples.

Table 4. Statistical summary of the simple linear regression.

\begin{tabular}{lccccc}
\hline & Estimado & Erro Padrão & Valor de t & P-Valor \\
\hline Intercepto & $-335,37$ & 49,04 & $-6,84$ & $6,18 \mathrm{E}-08$ & ${ }^{* * *}$ \\
P90 & 24,25 & 1,78 & 13,64 & $1,42 \mathrm{E}-15{ }^{* * *}$ \\
\hline
\end{tabular}

Obs.: **** significativo a $0,001 \%$.

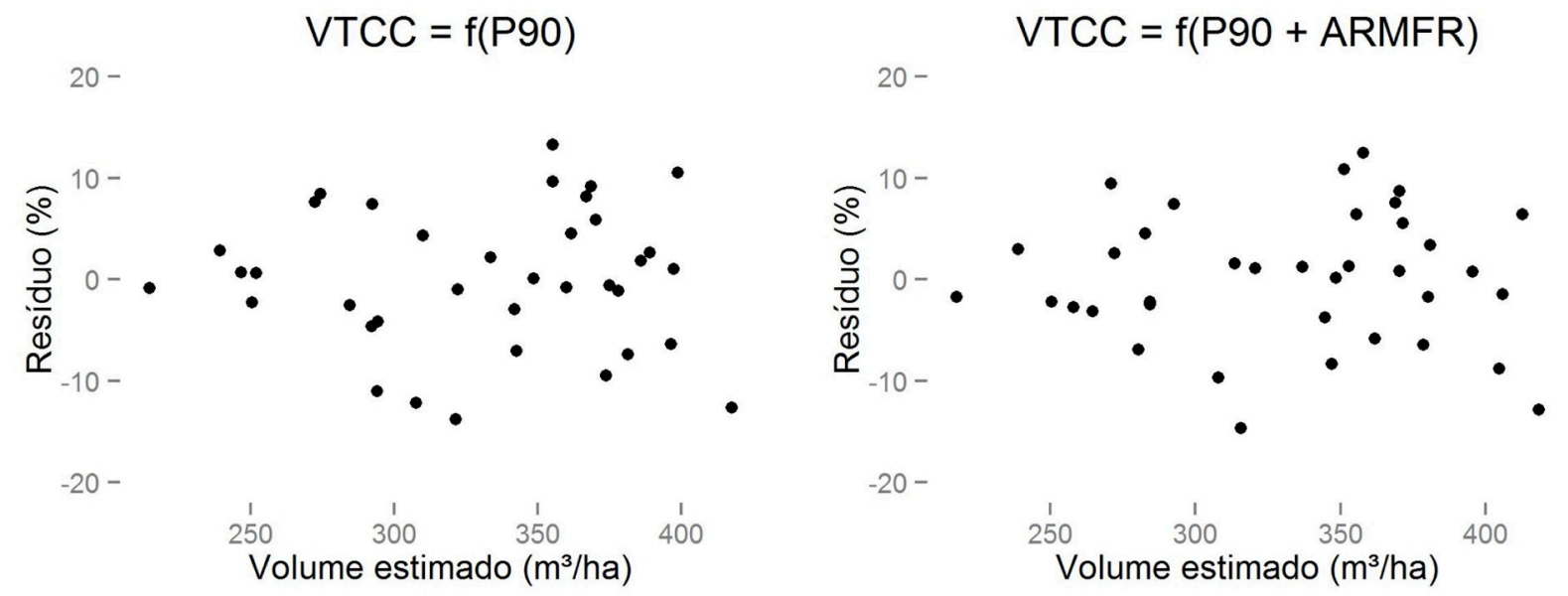

Figura 2. Gráfico percentual dos resíduos em função do volume estimado.

Figure 2. Percentage of the residuals related to the estimated volume.

A regressão linear múltipla usada na DA II (Equação 8) composta por uma métrica de altura (P90) e uma de densidade (ARMFR) apresentou coeficientes significativos (Tabela 5), coeficiente de determinação ajustado ( $\mathrm{R}^{2} \mathrm{adj}$ ) de 85,3 \% e erro padrão dos resíduos de 22,15 ( $\left.\mathrm{m}^{3} / \mathrm{ha}\right)$. A análise de resíduos pode ser visualizada na Figura 2. 
Tabela 5. Resumo estatístico da regressão linear múltipla.

Table 5. Statistical summary of the multiple linear regression.

\begin{tabular}{lccccc}
\hline & Estimado & Erro Padrão & Valor de t & \multicolumn{2}{c}{ P-Valor } \\
\hline Intercepto & $-435,34$ & 65,56 & $-6,64$ & $1,28 \mathrm{E}-07$ & $* * *$ \\
P90 & 24,37 & 1,69 & 14,41 & $4,88 \mathrm{E}-16$ & $* *$ \\
ARMFR & 1,59 & 0,73 & 2,17 & 0,0371 & $*$ \\
\hline
\end{tabular}

Obs.: *** significativo a $0,001 \%$ e * significativo a $0,05 \%$

A utilização da a alta correlação entre as métricas LiDAR (P90 e ARMFR) e os parâmetros populacionais do povoamento possibilitou uma estimativa por meio de dupla amostragem mais precisa da variável de interesse (volume). A redução do erro amostral por meio de DA pode reduzir custos, uma vez que menores intensidades amostrais podem manter o erro amostral dentro de intervalos satisfatórios. Stephens et al. (2011) também obtiveram melhora aplicando a dupla amostragem utilizando métricas LiDAR como primeira fase para estimar o estoque de carbono nas florestas da Nova Zelândia. A regressão linear simples utilizada vinculando a primeira e a segunda fase da dupla amostragem obteve um coeficiente de correlação $\left(\mathrm{R}^{2}\right)$ de $74 \%$, melhorando em $6 \%$ a estimativa anterior do estoque de carbono. Utilizando dados de biomassa de uma floresta simulada, Næsset et al. (2013) encontraram uma redução do erro padrão de $\pm 2,8 \mathrm{Mg} \mathrm{ha}^{-1}$, considerando a amostragem casual simples, para $\pm 1,0 \mathrm{Mg} \mathrm{ha}^{-1}$ na dupla amostragem utilizando dados LiDAR.

Na simulação desenvolvida para verificar a intensidade amostral para um erro aceitável de $\pm 5 \%$, a dupla amostragem permitiu uma redução de $71,4 \%$ do número de parcelas em relação à amostragem casual simples. Para um erro aceitável de $\pm 5 \%$, são necessárias 35 parcelas seguindo a ACS, 17 parcelas na ACE I. Na ACE II e na DA, a intensidade amostral atingiu o limite mínimo de parcelas proposto anteriormente sem que o erro atingisse os $\pm 5 \%$. O erro amostral da ACE II ficou em $\pm 3,5 \%$ (18 parcelas - 3 por extrato) e o erro amostral da DA I e da DA II ficaram em $\pm 3,8 \%$ (10 parcelas) e $\pm 3,4 \%$ (10 parcelas), respectivamente.

Tabela 6. Tamanho amostral dos delineamentos amostrais para o erro máximo de $\pm 5 \%$.

Table 6. Sample size of sampling designs for a maximum error of $\pm 5 \%$.

\begin{tabular}{ccc}
\hline Amostragem & Número de Parcelas & Erro Amostral (\%) \\
\hline ACS & 35 & $\pm 5,0$ \\
ACE I & 17 & $\pm 4,8$ \\
ACE II & 18 & $\pm 3,5$ \\
DA I & 10 & $\pm 3,8$ \\
DA II & 10 & $\pm 3,4$ \\
\hline
\end{tabular}

\section{CONCLUSÕES}

A alta correlação existente entre o volume e as métricas LiDAR permitiu uma considerável melhora das estimativas de volume no inventário florestal, através da aplicação da dupla amostragem. Seu uso permite inclusive uma redução do número de parcelas medidas em campo para um determinado erro amostral tolerável.

Com a popularização e redução dos custos associados aos levantamentos laser aerotransportados, o uso desta tecnologia pode trazer ganhos consideráveis de exatidão e de redução de custos. Especialmente para inventários de grandes áreas, inclusive considerando florestas naturais que se localizam em locais cujo trabalho de campo ocorre em condições extenuantes. Dessa forma, os inventários nacionais também podem se beneficiar de uma abordagem de dupla amostragem com primeira fase baseada em métricas LiDAR.

\section{AGRADECIMENTOS}

Este trabalho é parte do programa de pesquisa desenvolvido pelo GET-LiDAR (Grupo de Estudos em Tecnologia LiDAR) do Departamento de Ciências Florestais, ESALQ/USP. Agradecemos o apoio da Suzano Papel e Celulose por viabilizar o levantamento laser aerotransportado e pela coleta dos dados de campo. Agradecemos também à CAPES pelas bolsas de mestrado e doutorado concedidas aos pesquisadores envolvidos neste projeto. Agradecemos aos revisores anônimos pelas valiosas contribuições. 
Laranja et al. - Redução do erro amostral na estimativa do volume de povoamentos

de Eucalyptus ssp. por meio de escaneamento laser aerotransportado

\section{REFERÊNCIAS BIBLIOGRÁFICAS}

ANDERSEN, H. E.; BREIDENBACH, J. Statistical properties of mean stand biomass estimators in a Lidar-based double sampling forest survey design. In: ISPRS WORKSHOP ON LASER SCANNING 2007 AND SILVILASER 2007, 36, 2007, Espoo. Anais... Espoo: ISPRS, 2007. p. 8-13.

CAMPOS, J. C. C.; RIBEIRO, J. O.; PAULA NETO, F. Inventário florestal nacional: reflorestamento Minas Gerais. Brasília: IBDF, 1984. 126 p.

CESARO, A.; ENGEL, O. A.; FINGER, C. A. G.; SCHNEIDER, P. R. Comparação dos métodos de amostragem de área fixa, relascopia, e de seis árvores, quanto a eficiência, no inventário florestal de um povoamento de Pinus sp. Ciência Florestal, Santa Maria, v. 4, n. 1, p. 97-108, jun. 1994.

GAVA, J. L. Efeito da adubação potássica em plantios de E. grandis conduzidos em segunda rotação em solos com diferentes teores de potássio trocável. Série Técnica IPEF, Piracicaba, n. 30, p. 89-94, 1997.

GIONGO, M.; KOEHLER, H. S.; MACHADO, S. A.; KIRCHNER, F. F.; MARCHETTI, M. LiDAR: princípios e aplicações florestais. Pesquisa Florestal Brasileira, Colombo, v. 30, n. 63, p. 231-244, ago./out. 2010.

MALTAMO, M.; MUSTONEN, K.; HYYPPÄ, J.; PITKÄNEN, J.; YU, X. The accuracy of estimating individual tree variables with airborne laser scanning in a boreal nature reserve. Canadian Journal of Forest Research, Ottawa, v. 34, n. 9, p. 1791-1801, 2004.

NÆSSET, E.; GOBAKKEN, T.; BOLLANDSÅS, O. M.; GREGOIRE, T. G.; NELSON, R. STÅHL, G. Comparison of precision of biomass estimates in regional field sample surveys and airborne LiDAR-assisted surveys in Hedmark County, Norway. Remote Sensing of Environment, New York, v. 130, p. 108-120, mar. 2013.

PÉLLICO NETTO, S.; BRENA, D. A. Inventário Florestal. Curitiba: Editado pelos autores, 1997. 316 p.

SCHUMACHER, F. X.; HALL, F. S. Logarithmic expression of timber-tree volume. Journal of Agricultural Research, Washington, v. 47, n. 9, p. 719-734, 1933.

SHIVER, B. D.; BORDERS, B. E. Sampling techniques for forest resource inventory. New York: John Wiley \& Sons, $1996,356 \mathrm{p}$.

SILVA, A. G. P.; GÖRGENS, E. B.; CAMPOE, O. C.; ALVARES, C. A.; STAPE, J. L.; RODRIGUEZ, L. C. E. Assessing biomass based on canopy height profiles using airborne laser scanning data in eucalypt plantations. Scientia Agricola, v. 72, n. 6, p. 504-512, 2015.

STEPHENS, P. R.; KIMBERLEY, M. O.; BEETS, P. N.; PAUL, T. S. H.; SEARLES, N.; BELL, A.; BRACK, C.; BROADLEY, J. Airborne scanning LiDAR in a double sampling forest carbon inventory. Remote Sensing of Environment, New York, v. 117, p. 348-357, 2012.

VAN DEUSEN, P. C.; PRISLEY, S. P.; LUCIER, A. A. Adopting an annual inventory system: user perspectives. Journal of Forestry, Bethesda, v. 97, n. 12, p. 11-14, 1999.

ZONETE, M. F. Estimação de parâmetros biométricos de plantios clonais de eucalipto no sul da Bahia: uma aplicação da tecnologia laser aerotransportada. Scientia Forestalis, Piracicaba, v. 38, n. 86, p. 225-235, 2010. Disponível em: < http://ipef.br/publicacoes/scientia/nr86/cap10.pdf > . Acesso em: 23 mai. 2014.

Recebido em 22/10/2014

Aceito para publicação em 22/05/2015 